\title{
Proposição de design de serviços para uma biblioteca pública com uma abordagem de design centrado no usuário
}

Proposition of service design for a public library with a user-centered design approach

VAVOLIZZA, Renata; Mestra em Design; UFPR - Universidade Federal do Paraná renatavavolizza@gmail.com

RAMOS, Marcos Roberto; Doutorando em Design; UFSC - Universidade Federal de Santa Catarina

marcos.roberto@univali.br

ANDALÓ, Flávio; Mestre em Design; Universidade Federal de Santa Catarina

fla2@uol.com.br

RIASCOS, Carmen E. M.; Mestre em Energia; Universidade Federal de Santa Catarina carmen.elena88@gmail.com

MERINO, Giselle Schmidt Alves Díaz; Doutora; Universidade Federal de Santa Catarina giselle.merino@posgrad.ufsc.br

MERINO, Eugenio Andrés Díaz; Doutor; Universidade Federal de Santa Catarina eugenio.merino@ufsc.br

\section{Resumo}

As bibliotecas são reconhecidas por preservar e disponibilizar o conhecimento gerado pela humanidade. Este espaço arquiva documentos durante anos e proporciona o acesso à população, gerando um serviço prestado a uma determinada comunidade. O serviço disponibilizado é o foco deste estudo, com o objetivo de melhorar o projeto de serviços em uma Biblioteca Pública a partir de uma abordagem de Design Centrado no Usuário. Desenvolveu-se uma pesquisa aplicada, com abordagem qualitativa, objetivos exploratórios e métodos analíticos. Aplicou-se a metodologia projetual "Guia de Orientação para Desenvolvimento de Projetos - GODP" aliada aos procedimentos técnicos, destacando-se o instrumento de pesquisa eye tracking. Os resultados apontam que em virtude das diversas interações existentes em um espaço público, como este da biblioteca, é necessário acompanhar as mudanças sociais e comportamentais dos usuários, para que os serviços prestados sejam condizentes com as necessidades, ao longo do tempo.

Palavras Chave: design de serviço; design centrado no usuário; biblioteca e eye tracking.

\section{Abstract}

Libraries are recognized for preserving and making available the knowledge generated by humanity. This space shelves documents for years and provides access to the 
population, generating a service provided to a particular community. The service provided is the focus of this study, with the objective of improving the design of services in a Public Library from a User-Centered Design approach. An applied research was developed, with qualitative approach, exploratory objectives and analytical methods. Applying the project methodology "Guide of Guidance for Development of Projects - GODP" allied to the technical procedures, The results show that due to the diverse interactions in a public space, such as this one in the library, it is necessary to follow the social and behavioral changes of the users, so that the services provided are in line with the needs, over time.

Keywords: service design; user-centered design; libraries and eye tracking.

\title{
1. Introdução
}

Este artigo trata sobre uma pesquisa e proposição de Design de serviços para uma Biblioteca Universitária, da Universidade Federal de Santa Catarina, localizada na cidade de Florianópolis. A iniciativa utilizou uma abordagem de Design Centrado no Usuário para desenvolvê-lo projetualmente.

A biblioteca Universitária da Universidade Federal de Santa Catarina, conhecida também como BU, é um espaço público que atende alunos de diversos níveis desde as séries iniciais até níveis de pós-graduação. Este espaço também é aberto à Comunidade externa à Universidade. As principais atividades realizadas pelos colaboradores da BU são armazenar a produção acadêmica e disponibiliza-la aos interessados cadastrados. Esta produção pode apresentar-se como livros, revistas, dissertações, teses, de forma física ou virtual. A informação é concreta, porém não existe a posse do usuário sobre o material emprestado, caracterizando a prática do empréstimo como um processo, de repetição ao longo do tempo com diferentes atores envolvidos. 0 fato da atividade principal da Biblioteca Universitária permear a intangibilidade e repetir-se em níveis processuais, caracterizam a mesma como um serviço.

Para Kotler e Armstrong (1999) serviço é "toda atividade ou benefício, essencialmente intangível, que uma parte pode oferecer a outra e que não tenha como resultado a posse de bens". Essa visão é compartilhada por Shotstack (1984 apud PINE; GILMORE, 1998, p. 49) reforçando que os serviços são trocas invisíveis que acontecem em todos os lugares, sendo intangíveis e não permitindo a posse. Estão ligadas as experiências, que são pessoais e existem dentro da mente do indivíduo.

A pesquisa buscou em Design de serviços a fundamentação teórica para estruturála. Utilizou também uma abordagem de Design Centrado no humano, através da metodologia de projeto, para desenvolver uma proposta de serviço que contemplasse as pessoas que utilizam este serviço.

\begin{abstract}
0 design centrado no ser humano pode ser definido como o processo que coloca as necessidades e limitações humanas em uma prioridade mais alta em comparação com outras metas durante os estágios diferenciais do pensamento de projeto e da produção. Durante esse processo, o designer precisa não apenas analisar e encontrar soluções para os problemas existentes, mas também testar e validar os produtos ou serviços projetados para atingir as metas planejadas no mundo real. (ELMANSY, 2017)
\end{abstract}

O Design centrado no usuário/humano é uma abordagem, da qual se faz necessário um processo empático para descobrir as necessidades e reprojetá-las. 
Segundo Elmansy (2015) para alcançar a abordagem é preciso adotar princípios que coloquem o usuário ao centro, garantindo um estudo de como o ser humano interagirá com o produto/serviço. "Empatia é a arte de se colocar no lugar do outro por meio da imaginação, compreendendo seus sentimentos e perspectivas e usando essa compreensão para guiar as próprias ações". (KRZNARIC, 2015).

Donald Normann (1986) cunhou o termo Design centrado no usuário, através do livro de sua autoria 'Design de Sistema Centrado no Usuário: novas Perspectivas na Interação Humano-Computador', posteriormente originou-se o termo Design centrado no humano.

Este estudo possui os seguintes objetivos:

1. Propor um Design de serviços para a Biblioteca Universitária da Universidade Federal de Santa Catarina.

2. Aplicar o Guia de Orientação para Desenvolvimento de Produtos - GODP, modelo de pesquisa e metodologia em Design centrado no usuário, nas fases de inspiração e ideação.

3. Aplicar como suporte de pesquisa ferramentas de design, no contexto do Design de serviços e Design centrado no usuário/humano, sendo elas: jornada do usuário e eyetracking.

4. Verificar a eficácia dos métodos empregados para coletar dados, registrar e apresentar a iniciativa para demais pesquisadores.

A primeira parte do artigo apresentará a Visão metodológica; posteriormente a Fundamentação teórica, apresentando o conceito de Design de serviços; seguirá com a Aplicação da metodologia de projeto GODP, referentes a inspiração e ideação; apresentará os Resultados e concluirá com as Considerações Finais.

\section{Visão metodológica da pesquisa}

O método escolhido para o desenvolvimento desta pesquisa visa atingir os objetivos explicitados neste documento. A construção está dividida em duas macros fases.

A primeira fase é a investigação por fundamentos de pesquisa e levantamento de dados para embasamento teórico. Utilizou-se procedimentos técnicos como revisão bibliográfica e pesquisa documental a arquivos públicos. Na segunda fase, houve uma aplicação de método projetual em Design centrado no usuário, para compreender a oportunidade/problema e oferecer uma proposta de design de serviços. Trabalhou-se com a metodologia GODP - Guia de Orientação para Desenvolvimento de Projetos, possibilitando a caracterização do objeto de pesquisa, identificação e visualização do mesmo a partir de visitas inloco e utilização da observação sistemática, jornada do usuário e eyetracking.

Segundo Demo (2000), as pesquisas variam conforme seus gêneros: teórica, dedicada a estudar teorias; metodológica, que se ocupa dos modos de se fazer ciência; empírica, dedicada a codificar a face mensurável da realidade social; prática, voltada para intervir na realidade social.

Esta pesquisa é prática de natureza aplicada, pois “...objetiva gerar 
conhecimentos para aplicação prática dirigidos à solução de problemas específicos. Envolve verdades e interesses locais." (PRODANOV, 2013).

A pesquisa apresenta ainda uma abordagem qualitativa, Segundo Yin (2010) a abordagem qualitativa gera significado atribuído aos fatos - exploração, observação e interpretação. Possui objetivos exploratórios, pois busca diferentes enfoques para o tema, permitindo uma visão sob diversos ângulos.

Com relação aos procedimentos, é uma pesquisa bibliográfica e documental, mas também uma pesquisa-ação. Possui os seguintes aspectos que segundo Thiollent (1998) a caracterizam: há ampla e explícita interação entre pesquisadores e pessoas implicadas na situação investigada; dessa interação resulta a ordem de prioridade dos problemas a serem encaminhados sob forma de ação concreta; o objeto de investigação não é constituído pelas pessoas e sim pela situação social e pelos problemas de diferentes naturezas encontrados nessa situação. A pesquisa-ação consiste em resolver ou pelo menos esclarecer os problemas da situação observada; a pesquisa não se limita a uma forma de ação (risco de ativismo), pretende-se aumentar o conhecimento dos pesquisadores e o conhecimento ou o 'nível de consciência' das pessoas e dos grupos considerados.

O Quadro 1 oferece a representação esquemática da classificação desta pesquisa.

\begin{tabular}{|c|c|c|}
\hline \multicolumn{3}{|c|}{ CLASSIFICAÇÃO DA PESQUISA } \\
\hline Categorias de classificação & Classificação desta pesquisa & Classificações existentes \\
\hline 1. Natureza & - Aplicada & $\begin{array}{ll}\text { - } & \text { Aplicada } \\
\text { Básica }\end{array}$ \\
\hline 2. Objetivos & - Exploratória & $\begin{array}{ll}\text { - } & \text { Descritiva } \\
\text { - } & \text { Explicativa } \\
\text { Exploratória }\end{array}$ \\
\hline 3. Abordagem & - Qualitativa & $\begin{array}{l}\text { - } \quad \text { Qualitativa } \\
\text { - }\end{array}$ \\
\hline 4. Procedimentos técnicos & $\begin{array}{l}\text { Bibliográfica } \\
\text { - }\end{array}$ & $\begin{array}{ll}: & \text { Bibliográfica } \\
: & \text { Documental } \\
: & \text { Surves-facto } \\
: & \text { Participante } \\
: & \text { Pesquisa-ação } \\
\text { Entre outros }\end{array}$ \\
\hline
\end{tabular}

Quadro 1 - Classificação da pesquisa.

Fonte: elaborado pelos autores, com base na pesquisa realizada.

\section{Design de serviços}

O design, enquanto atividade multidisciplinar, é visto cada vez mais, como uma atitude, uma relação entre pensar e fazer, passível de aplicação em diferentes tipos de produtos, ou seja, objetos, sinais, interações, serviços e sistemas. 0 desenvolvimento recente do design de serviços, como abordagem ou profissão, espelha essa evolução, contribuindo para uma mudança de contexto, concepção de melhores interações de 
serviços e experiências, aplicando ferramentas oriundos do design de experiência (MARGER, 2007).

Nesse contexto, o design de serviços surge carregando consigo um conjunto de áreas de conhecimento, métodos e práticas que permitem que equipes multidisciplinares trabalhem na construção de ofertas de serviços mais adaptadas às pessoas (PINHEIRO, 2014). Do mesmo modo, o designer vê-se como um projetista de melhores soluções na disponibilidade desses serviços, ampliando a satisfação do usuário.

A evolução da pesquisa em design de serviços vem se desenvolvendo em diferentes escolas de pensamento ou em campo transdisciplinar. Entre 2008 e 2009 surgiram novos pesquisadores publicando trabalhos sobre design de serviços, assim como novas publicações acadêmicas. Em vez de tentar justificar o design de serviços como disciplina, os textos passaram a abordar a pesquisa em design de serviços (STICKDORN; SCHNEIDER, 2014). O mesmo autor ainda observa que boa parte das primeiras pesquisas tinham como foco a conexão da área com outras disciplinas e sua defesa como disciplina autônoma. Os primeiros pesquisadores tinham formações em outras áreas e sua transição para a área de design de serviços foi gradual (STICKDORN; SCHNEIDER, 2014).

Design de serviços é um tema que demanda ser estudado com maior abrangência e aprofundamento. Existe uma crescente importância do setor de serviços para a economia global. A forma pela qual organizações podem criar conhecimento a partir de redes de valor para a geração de novos serviços é o que definem Lovelock e Wright (2003) como "um ato ou desempenho oferecido por uma parte à outra".

Segundo Mager (2009), serviços fornecem um conjunto de ferramentas que "estimulam designers de serviços a criar interações, espaços e processos com base em um conhecimento sólido a respeito de determinadas relações causais". Aborda a funcionalidade e a forma de serviços a partir da perspectiva dos clientes. 0 objetivo é garantir que as interfaces do serviço sejam úteis, utilizáveis e desejáveis do ponto de vista do cliente e eficazes, eficientes e diferenciadas do ponto de vista do fornecedor (BLOMKVIST et al. apud STICKDORN; SCHNEIDER, 2014).

Novas tecnologias utilizadas pela sociedade, voltadas para uma melhoria na qualidade de vida, foram projetadas e organizadas de forma a melhorar as relações sociais, projetando o design de serviços como uma necessidade de se estabelecer uma visão mais humana aos serviços (LANGENBACH, 2008).

O primeiro passo no conceito de serviços é repensar o próprio serviço. Uma abordagem do design de serviços é o conceito de serviço, sendo possível projetar serviços mais humanos, valorizando a relação serviço-ser-humano. Por isso, Manzini (2007) aborda essa questão como uma mudança no nosso ponto de vista, saindo cada vez mais dos objetos para os serviços, e nos serviços buscando a essência deles. Em muitos casos, o projeto e o desenvolvimento de um produto não condiz com a qualidade que 0 serviço oferecido na sua interação é prestado, e vice-versa. Quando essa relação não é coerente, a relação do cliente com a empresa se enfraquece.

A natureza do serviço deve estar clara e ser compartilhada por todos os envolvidos no serviço. Nessa mesma perspectiva, Goldstein et al. (2002) pensa na desconstrução do serviço por meio das perguntas de como funciona e do que ele é, e da integração desses dois fatores, ou ainda por meio de seus componentes, possibilitando identificar os vários elementos do conceito do serviço, checando em relação às necessidades dos clientes e redesenhando esses elementos, entendendo o conceito do serviço como uma 
experiência.

Em síntese, design de serviços inverte o olhar do designer como desenvolvedor de produto e amplia sua visão para projetar como o cliente ou o usuário irá recebê-lo, quais possíveis dificuldades necessitam ser atendidas e como será possível tornar essa experiência mais interessante possível.

\section{Aplicação do Guia de Orientação para Desenvolvimento de Projetos - GODP}

0 modelo aplicado possui autoria de Merino ${ }^{1}$, trata-se de uma metodologia de design centrado no usuário, com propósito de difundir práticas projetais em design com foco no ser humano. Atinge seu objetivo através do apoio à investigação científica unida a essência do ponto central que rege o design: a atividade projetual.

Merino (2016) salienta que a não aceitação de projetos em geral dá-se devido o conhecimento insuficiente sobre as pessoas. Portanto o GODP é uma metodologia que auxilia na compreensão de grande quantidade de informação, sempre colocando em voga o usuário, seu contexto e produto a ser projetado para este ser humano.

Neste pesquisa, o desafio é aplicar o Guia em um contexto de serviços. O serviço lida fortemente com a experiência. Podemos ter diferentes experiências e algumas podem ser projetadas. A experiência é motivo de estudo e projeto tanto para o Design de Serviços como para o Design Centrado no Usuário.

Quando nos referimos a Projeto Centrado no Usuário, tratamos das capacidades humanas: sensorial (visão, audição, tato, olfato e paladar) cognitiva (pensamento e comunicação) e motora (alcance, locomoção e destreza). Juntamente com as dimensões temporal (nascemos, crescemos e envelhecemos) e social (aspectos culturais e de contexto), configurando um processo altamente empático. (MERINO, 2016)

O guia aplicado apresenta um formato para oito etapas de projeto, permitindo utilizar ferramentas conjuntamente, para construir uma rede de informações que vão oferecendo estrutura ao projeto.

O objetivo do guia é o de organizar e oferecer uma sequência de ações que permitam com que o Design seja concebido de forma consciente, levando em consideração o maior número de aspectos, e respondendo de forma positiva e consistente aos objetivos fixados para o projeto. (MERINO, 2016)

${ }^{1}$ Giselle Schmidt Alves Díaz Merino é professora permanente do Programa de Pós-graduação em Design da Universidade Federal de Santa Catarina (Florianópolis). Pós Doutora em Fatores Humanos pela Universidade do Estado de Santa Catarina. Doutora em Engenharia de Produção pela Universidade Federal de Santa Catarina (2014). Mestra em Design pela Universidade Federal de Santa Catarina (2010). Graduada em Desenho pela Universidade do Estado de Santa Catarina (1997). 


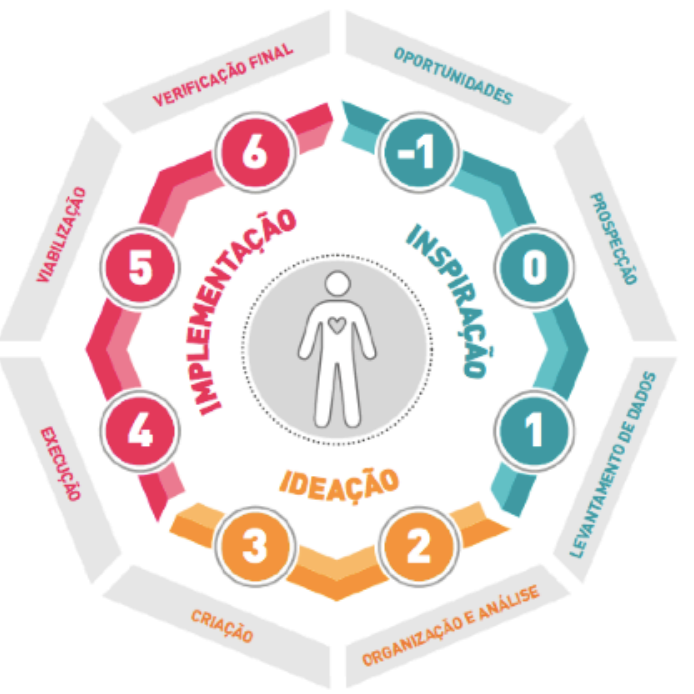

Fonte: Merino (2016)

O Guia utiliza embasamento nas grande fases projetuais propostas por Brown, (2009), através do Design Thinking: inspiração, ideação e implementação. Cada fase é subdividida, gerando um total de 08 etapas, identificadas de -1 a 6 . 0 projeto da Biblioteca Universitária utilizou 05 etapas, identificadas até o número 3 , momento criação deste mapa. Logo os resultados deste artigo tangem até a última etapa desenvolvida pelos autores. 0 quadro 2 demonstra de forma esquemática a metodologia aplicada.

Quadro 2 - Aplicação da metodologia estudada.

\begin{tabular}{|l|l|}
\hline \multicolumn{2}{|c|}{ GODP } \\
\hline \multicolumn{2}{|c|}{ Guia de Orientação para Desenvolvimento de Projetos } \\
\hline \multicolumn{1}{|c|}{ Etapas de pesquisa do método } & $\begin{array}{l}\text { Informações coletadas/trabalhadas - Biblioteca } \\
\text { Universitária }\end{array}$ \\
$\begin{array}{l}\text { 1. Oportunidades } \\
\text { Identificação de oportunidades de } \\
\text { mercado. }\end{array}$ & - $\begin{array}{l}\text { Levantamento de serviços a serem } \\
\text { projetados na Universidade. } \\
\text { Verificação do tempo disponível. } \\
\text { Observações: } \\
\text { Definição do objeto de estudo. }\end{array}$ \\
\hline
\end{tabular}




\section{Prospecção}

Identificação da demanda/problemática central que norteará o projeto.

\section{Observações:}

Realização de pesquisas preliminares para determinar ferramentas e preparação para coleta de dados.
- Levantamento de dados em meios digitais.

- Análise sincrônica.

- Análise diacrônica.

- Análise das fotografias antigas da BU.

\section{Levantamento de dados}

Entrevista semi estruturada para ser realizada com integrante do movimento.

\section{Observações:}

Realização de 150 fotografias da jornada do usuário. Utilização do eyetracking com captação de vídeo ao longo de todo processo, aproximadamente $1 \mathrm{~h} 30$.
- Formulação das tarefas a serem cumpridas pelo usuário.

- Preparação da observação sistemática baseada nas tarefas.

- Preparação do eyetracking.

- Visita ao local.

\section{Ideação}

Organização e análise de dados para definir as estratégias de projeto.

\section{Observações:}

Análise de todo material coletado e realização da jornada do usuário na planta atual.
- Análise dos vídeos gerados pelo eyetracking.

- Análise das fotografias geradas na jornada.

- Análise da planta baixa da biblioteca.

- Análise dos serviços prestados.

- Realização da jornada atual do usuário na planta baixa para gerar uma ideia espacial.

\section{Criação}

Geração de conceitos e alternativas de projeto. (Ao final é escolhida a alternativa que melhor responde especificações e objetivos de projeto.)

\section{Observações:}

Geração de um projeto de serviços para a BU da UFSC.
- Realização da jornada do usuário antiga em camadas.

- Realização da jornada do usuário nova em camadas.

- Nova proposição de serviços.

- Nova proposição de layout com base em um novo projeto de serviços.

Fonte: realizado pelos autores, com base no guia GODP.

\section{Resultados}

O início do projeto deu-se com a definição dos Blocos de Referências, propostos pelo guia, especificados como: produto, usuário e contexto.

Figura 2 - Blocos de Referência GODP
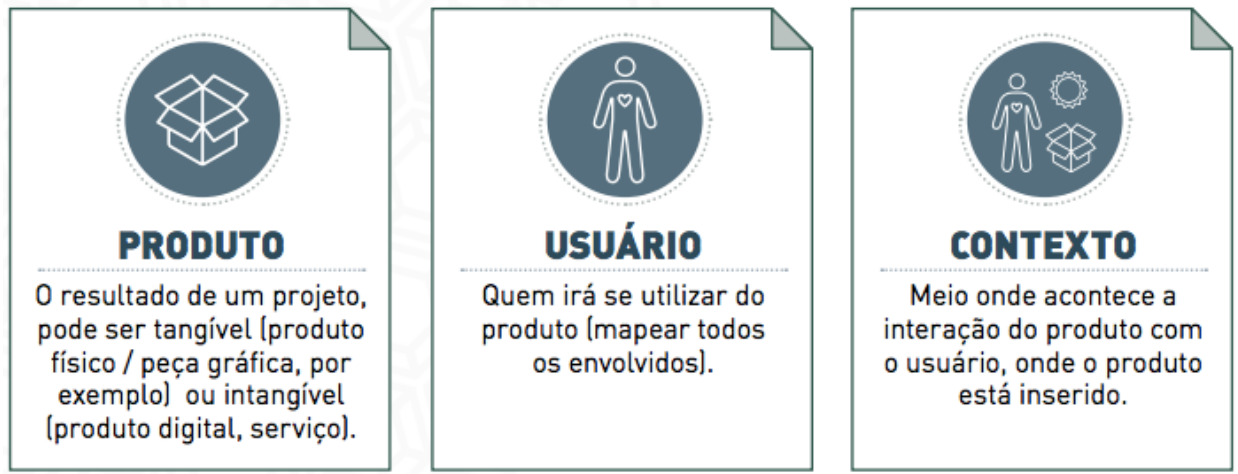
A cada Bloco de Referência, trabalhou-se uma ferramenta a qual poderia ampliar a compreensão da mesma, entendendo que o resultado seria oferecido em síntese, através de uma representação visual.

\subsection{Contexto}

Para o Bloco de Referência contexto, definiu-se a Pesquisa documental, assim como a Análise sincrônica e diacrônica como ferramentas para melhor compreensão do mesmo.

A análise diacrônica deu a base inicial para o projeto. Refere-se a um estudo de variações que coexistem numa determinada época, como regionais, sociais ou situacionais.

A Biblioteca Universitária da Universidade Federal de Santa Catarina iniciou suas atividades em 1968, porém seu prédio foi inaugurado somente em 1976. Passou a ter uma organização estruturada, assim como a denominação de BU a partir de 1976.

Em 1978 passa a gerar prestação de serviços e integração ao Centro Latinoamericano e do Caribe de Informação de Ciências da Saúde. Acompanhando a expansão da Universidade, a Biblioteca passa a ter o Sistema de Bibliotecas da UFSC, destinado a integrar e monitorar todo acervo existente nas bibliotecas setoriais, distanciadas geograficamente da BU.

A partir de 1996 o prédio principal da biblioteca é ampliado modificando não só a estrutura arquitetônica, mas também a estrutura de atendimento. Os anos seguintes foram marcados pela tecnologia da informação e expansão do acervo.

A informação sobre a ampliação trouxe consigo o questionamento sobre como era a BU anteriormente, levando os pesquisadores uma fotografia que gerou o início de construção dos resultados.

Figura 3 - BU da UFSC nos anos 70

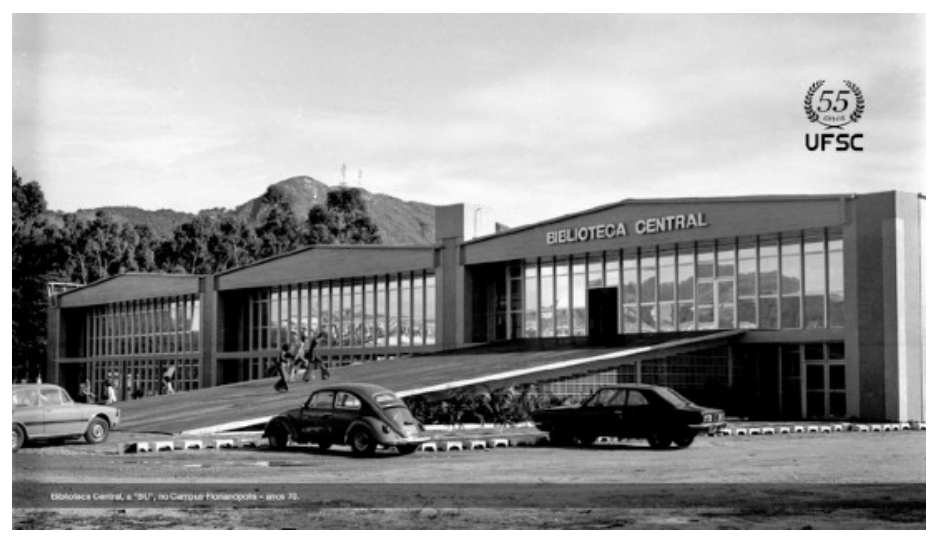

Fonte: Acervo documental da Biblioteca Universitária da UFSC.

Nota-se que existe uma rampa frontal, de acesso principal a biblioteca. Esta rampa levava o usuário diretamente ao atendimento. Fazendo com que todo acervo estivesse no segundo andar. Atualmente o usuário entra pelo primeiro andar na 
Biblioteca, porém passa por uma série de 'caminhos' até chegar ao objetivo principal do serviço prestado pela BU: consultar e retirar o material bibliográfico, que persiste no segundo andar.

Chega-se a conclusão que a ampliação considerou a arquitetura e engenharia envolvidas no prédio. Mas desconsiderou o serviço e a possibilidade de reprojetá-lo, destinado ao design. Um grande projeto como a $\mathrm{BU}$, necessita que diferentes áreas do conhecimento dialoguem para gerar um resultado satisfatório ao usuário.

Estas informações foram primordiais para a continuação do projeto, levando-se a Análise anterior como alimento para a construção do próximo bloco de referência.

\subsection{Usuário}

Para o Bloco de Referência usuário, definiu-se a observação sistemática, evidenciada com registro fotográfico e o eye tracking, ou rastreamento ocular como instrumento de levantamento de dados e análise de usabilidade e experiência do usuário para o processo de criação.

O Eye tracking é uma tecnologia para medir e gravar em tempo real as respostas fisiológicas aos estímulos visuais (ANDRIENKO et al., 2012; CHANDRA et al., 2015; DEDKOVA e POPELKA, 2015). A tecnologia do Eye tracking é baseada nos princípios de rastrear os movimentos do olho humano ao perceber a cena visual. 0 dispositivo utilizado para medir os movimentos oculares é comumente conhecido como um eyetracker e trata-se de um dos métodos de estudos de usabilidade e experiência do usuário considerado como não-tendencioso, porque não é influenciado pela opinião dos participantes como os outros métodos, tais como questionários ou entrevistas (DEDKOVA e POPELKA, 2015).

Os sistemas de Eye tracking recolhem os movimentos oculares durante 0 acompanhamento da emoção, leitura, reconhecimento de atividade humana percepção de publicidade, realizando uso de um site, interação humano-computador, sistema de auxílio na condução, cognição do esporte, sistemas de detecção da fadiga para pilotos, etc. (ANDRIENKO et al., 2012; CHANDRA et al., 2015). Também tem sido utilizado no processo de verificação da eficácia de treinamento (CARROLL et al., 2013; HASANZADEH et al., 2017). Da mesma forma, eye tracking tem sido usado para medir a satisfação dos usuários em testes de usabilidade (GOBBI et al., 2017) e para medir a usabilidade do usuário do dispositivo LEV (SCHMIDT et al., 2016).

O sistema compreende a um óculos equipado com uma câmera, para registrar o que o usuário está visualizando, e sensores infravermelhos que captam os movimentos dos olhos. Estes registros são transmitidos a um computador ou dispositivo móvel que sincroniza as informações coletadas permitindo a análise de onde o usuário estava efetivamente olhando, permitindo gerar diversos tipos de relatórios após o processamento dos dados.

Para essa pesquisa foi utilizado os óculos Eye Tracking Glasses $2 w$ da empresa alemã SMI (SensoMotoric Instruments), acompanhado do software BeeGaze fornecido pela mesma empresa. "O eye tracker vestível da SMI é a ferramenta de eye tracking mais reconhecida disponível para uso professional em pesquisas de marketing, fatores humanos, treinamento profissional e ciência cognitiva" (BUSINESS WIRE, 2014, tradução nossa). A aquisição dos dados pode ser feita utilizando os óculos conectados a um computador ou a um dispositivo móvel. Como a coleta de dados feita nessa pesquisa 
consistia na trajetória de um usuário pela biblioteca universitária da UFSC, utilizou-se um aparelho celular utilizando sistema Android equipado com o software disponível pela SMI de forma que o usuário pode mover-se livremente pelos ambientes ao mesmo tempo em que os dados eram coletados.

Figura 4 - exemplo de óculos com sistema de eye tracking.

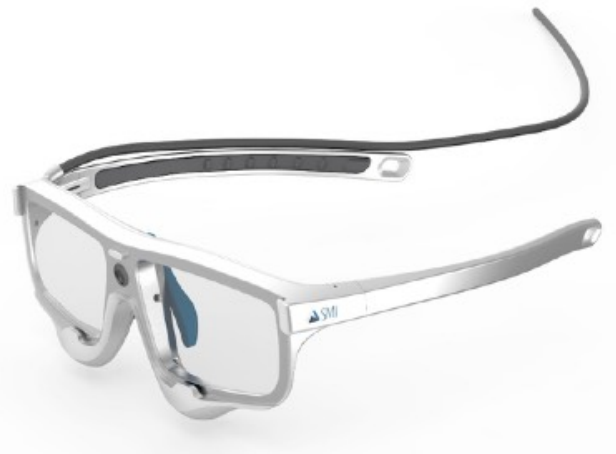

Fonte: BUSINESS WIRE (2014).

A coleta de dados, com o sistema utilizado nessa pesquisa, iniciou com o usuário colocando os óculos e conectando-o via cabo USB ao aparelho celular, utilizado exclusivamente para esta coleta. Outros celulares foram utilizados para filmagem e registro fotográfico nesta etapa da pesquisa. Antes de iniciar a coleta é necessário fazer uma calibragem no software de eye track, feita com o usuário olhando fixamente para um ponto e marcando esse ponto na tela do celular. Essa marcação do ponto deve ser feita três vezes, em três pontos diferentes para que o sistema esteja calibrado. Com isso pode-se iniciar a coleta de dados enquanto o usuário segue a jornada pré-determinada, observando sempre se o software está funcionando corretamente pois ocorreram diversas interrupções na captura, algumas permitindo a retomada logo em seguida e outras necessitando reiniciar o software e repetir a calibração.

Os dados obtidos foram transferidos do aparelho celular para um computador, onde é realizada a análise dos dados. 0 dado primário resultante é um vídeo captado pela câmera frontal mostrando a visão do usuário, acompanhado de um ponto que se movimenta indicando o local onde os olhos estão apontando. É possível gerar diversos relatórios como o mapa de calor, indicando as áreas mais vistas, ou mapa de fixação, indicando pontos em que os olhos ficam relativamente imóveis, fixos em um determinado ponto. A fixação pode ser representada pela quantidade, tempo de fixação ou dispersão, quando os olhos se afastam da área de interesse. Para que esses mapas possam ser gerados é necessário fazer uma correspondência entre os pontos para onde o olho estava apontando e os pontos na foto capturado no local. Esse processo é bastante demorado pois essa marcação deve ser feita manualmente, portanto uma jornada longa resulta em um longo tempo de trabalho antes de se obter os mapas para a análise.

A observação sistemática consistiu em instrumentar uma das pesquisadoras com o eye tracking e oferecer cinco ações rotineiras ao público da BU.

- Ação 01: entrar na biblioteca com seu material de estudo (inclusive notebook)

- Ação 02: efetuar cadastro 
- Ação 03: retirar 3 livros e 1 Dissertação

- Ação 04: avaliar o material utilizando as acomodações disponíveis no ambiente.

- Ação 05: sair da biblioteca.

A Figura 4 representa o primeiro contato visual do usuário ao entrar na BU. A análise demonstra a dispersão visual em busca de informações sobre 'entrar na biblioteca” , em função da profusão visual de elementos distribuídos sem hierarquia.

Figura 4 - imagens captadas com o sistema eye tracking.
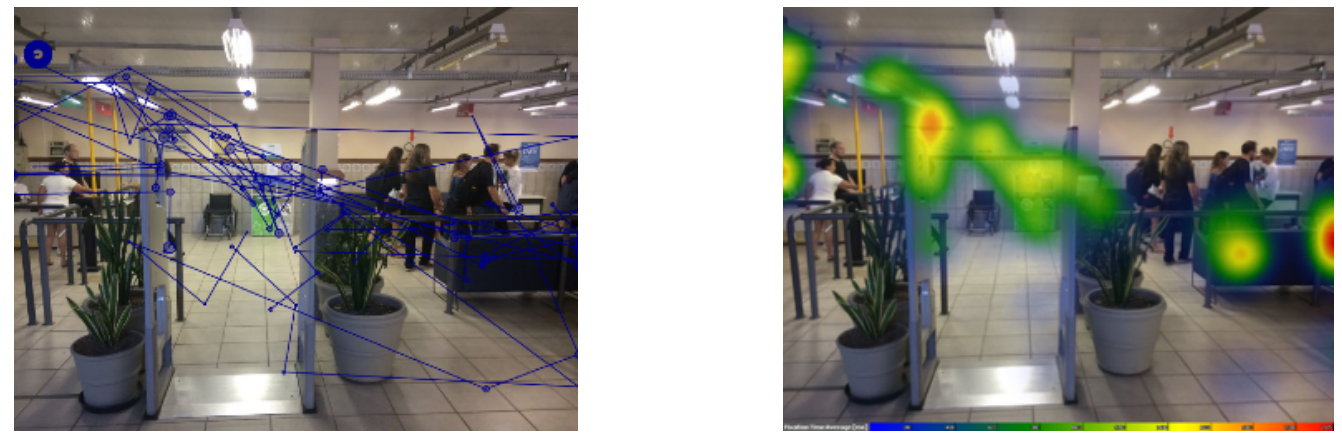

Fonte: elaborado pelos autores, com base na pesquisa realizada (2017).

A Figura 5 demonstra que após guardar seus pertences, a usuária foca seu olhar a atendente, ponto azul ou vermelho de maior tamanho na Figura 5, pois encontra novas barreiras para entrar na Biblioteca, impedindo que compreenda sua jornada de forma intuitiva.

Figura 5 - imagens captadas com o sistema eye tracking.
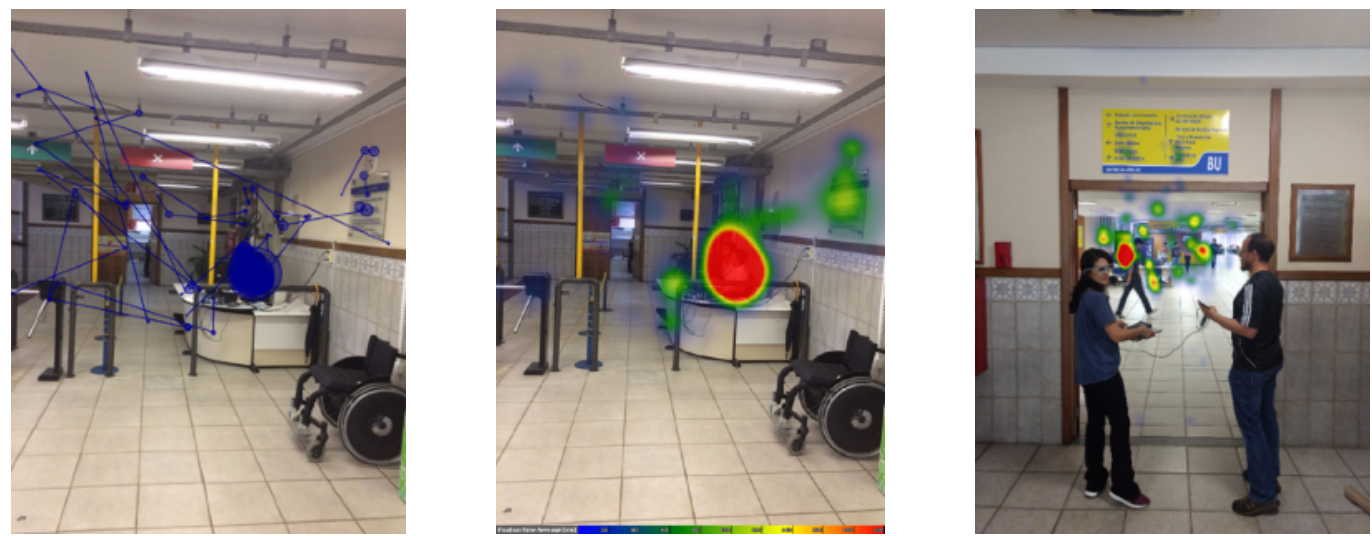

Fonte: elaborado pelos autores, com base na pesquisa realizada (2017).

As demais análises da observação sistemática com o eye tracking colaboraram para reforçar a necessidade de um projeto de serviço na BU, com enfoque nas pessoas. 


\subsection{Produto}

O Bloco de Referência produto, refere-se ao projeto de serviço proposto a BU da UFSC. A jornada do usuário em camadas foi eleita como a ferramenta de design escolhida para o processo de criação.

Todo o caminho percorrido pelo usuário com o eye tracking, para realizar a consulta e empréstimo bibliográfico é entendido como sua jornada ao longo do serviço, tal processo foi compreendido em camadas:

- Estágios: fases nos ambientes que se fazia necessário passar para conseguir efetivar o serviço. A cada estágio foi atribuído uma cor, para melhor interpretação.

Figura 6 - imagens captadas com o sistema eye tracking.

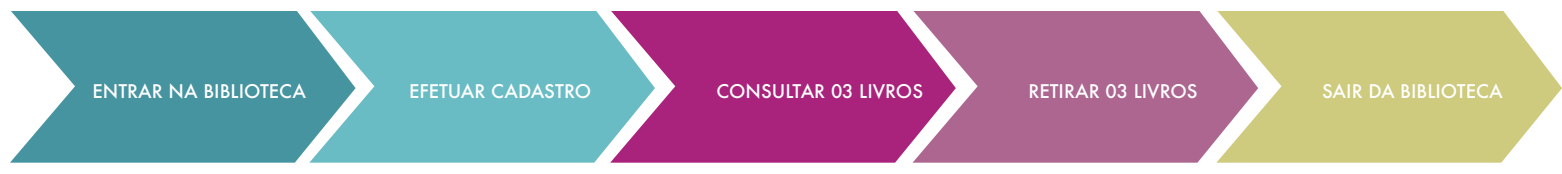

Fonte: elaborado pelos autores, com base na pesquisa realizada (2017).

- Ações: interação do usuário ao longo do serviço, que estão presentes em todos os estágios, conforme a cor atribuída.

Figura 7 - imagens captadas com o sistema eye tracking.
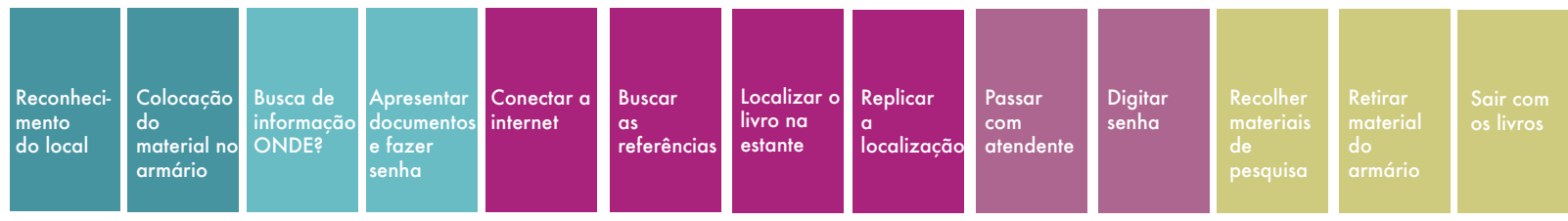

Fonte: elaborado pelos autores, com base na pesquisa realizada (2017).

- Pontos de contato: refere-se aos elemento físico ou digital do serviço com que o usuário entra em contato durante a experiência.

Figura 8 - imagens captadas com o sistema eye tracking.

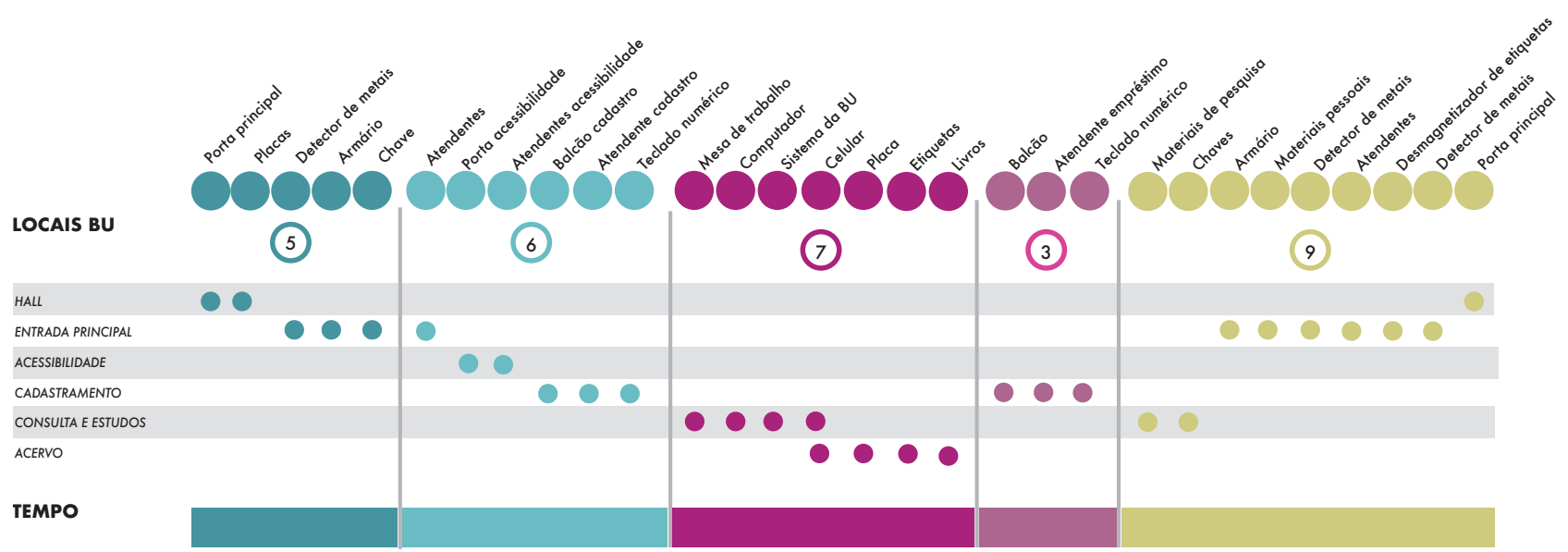


Fonte: elaborado pelos autores, com base na pesquisa realizada (2017).

Com base neste estudo, os pesquisadores propuseram uma solução que contempla a mudança do andar de atendimento, passando a ser exclusivamente no primeiro piso. Deixando o segundo andar somente para serviços internos. Esta mudança reduz sensivelmente os pontos de contato listados na jornada do usuário e efetiva a compreensão do espaço pelo público, gerando: fluxo no serviço; circulação de pessoas; saídas para evacuação; área verde; aumento de pontos de atendimento; e sinalização intuitiva.

A solução oferece ainda:

- Painel multimídia - inexistente na situação atual.

- Transportador de livros - inexistente na situação atual.

- 02 áreas de armários - atualmente possui somente uma disponível.

- 02 Saídas - atualmente possui somente uma e não há saída de emergência.

- 02 áreas de auto atendimento - atualmente existe somente uma.

- Muitas tomadas - número de tomadas escassas na situação atual.

- Área de exposição - realizada de forma vernacular e desorganizada.

- Diferentes tipos de acento - atualmente existem somente dois: cadeiras ou pufs.

- Barreira sonora - a sonorização não é trabalhada na situação atual.

- Aproveitamento da luz natural - atualmente existe utilização da luz artificial.

Figura 9 - novo layout, contemplando uma proposta de design de serviços
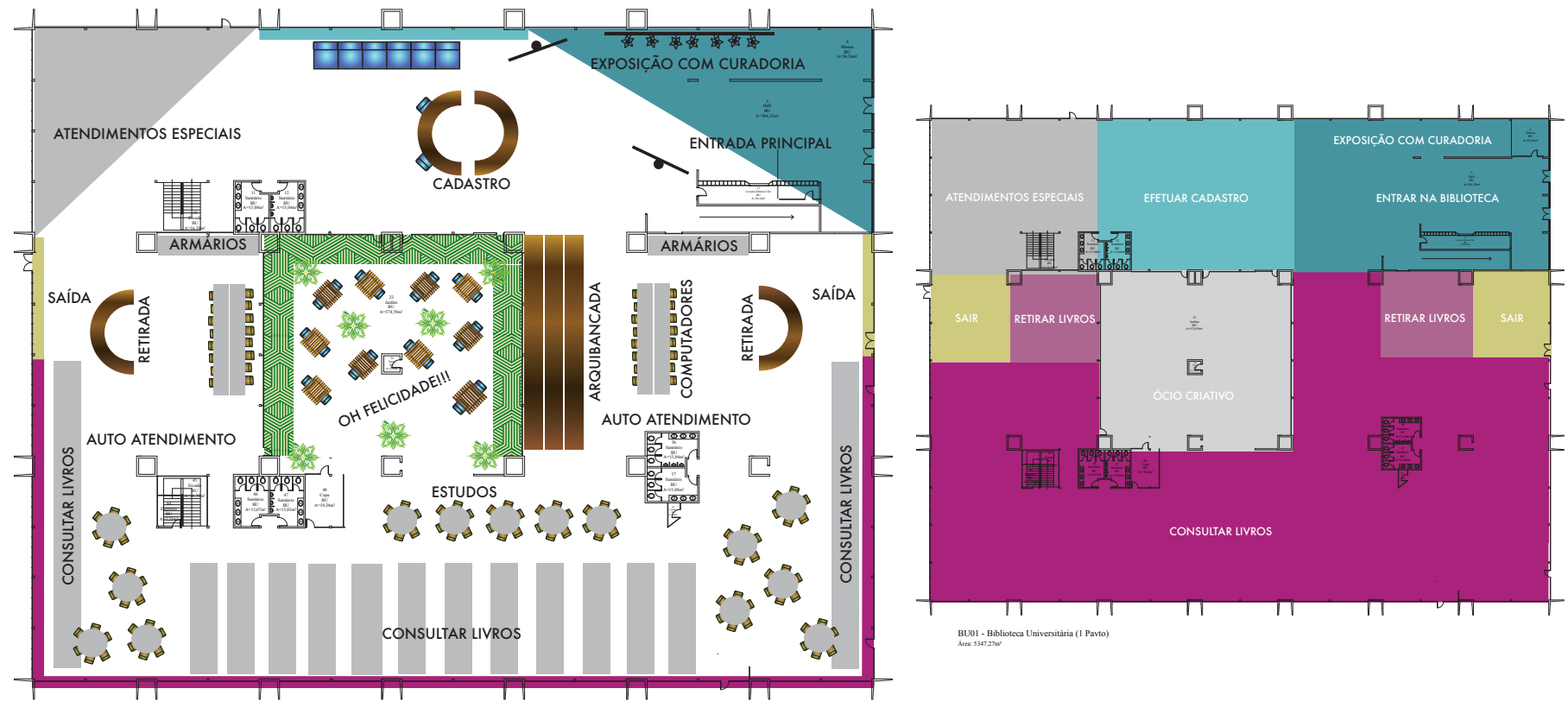

BU01- B Biblioceca Universitiria ( P Pavo

Fonte: elaborado pelos autores, com base na pesquisa realizada (2017). 


\section{Considerações finais}

O presente artigo objetivou aplicar uma metodologia projetual de Design Centrado no Usuário - GODP, para gerar um projeto de serviços, proposto a Biblioteca Universitária da UFSC.

Os resultados alcançados contemplaram o entendimento do contexto, a jornada do usuário e uma proposição em serviço, orientada pelo design. Estes dados são de grande importância para a comunidade acadêmica e para a sociedade, devido a conscientização do planejamento e projeto de espaços públicos que contém o capital intelectual de uma comunidade. 0 serviço prestado atualmente impacta diretamente na experiência do usuário, sendo passível de projetarão, conforme demonstrado neste artigo.

A capacidade multidisciplinar do designer permite sua atuação na educação, contribuindo para a organização espacial, comunicação visual, escolha de produtos e materiais adequados para os usuários realizarem determinadas tarefas em um serviço.

As cinco fases do Guia foram executadas com sucesso, demonstrando sua eficácia. Percebeu-se a importância de uma preparação de pesquisa planejada e a escolha de ferramentas adequadas para que a análise seja contundente.

Nota-se que em Design de serviços o elemento desafiador consiste em compreender as interações intangíveis para propor soluções tangíveis. Ainda assim, grande parte das soluções materializam-se pela mudança de perspectiva e não necessariamente pela posse ou aquisições dispersas. Por isso, faz-se necessário projetar o intangível para adquirir o tangível, um ponto de vista bastante diferente do praticado nas instituições públicas do Brasil.

Os pesquisadores vislumbram apresentar a proposta para os responsáveis da BU, não se acredita na possibilidade de finalização do projeto, mas sim na importância da conscientização das pessoas que oferecem tal serviço.

\section{Referências}

ANDRIENKO, G.; ANDRIENKO, N.; BURCH, M.; WEISKOPF, D. Visual analytics methodology for eye movement studies. IEEE Transactions on Visualization and Computer Graphics, 18(12), 28892898, 2012. https://doi.org/10.1109/TVCG.2012.276

BUSINESS WIRE. SMI Eye Tracking Glasses Set the Industry Standard for Professional Research and Training. In: Business Wire, 2014. (https://www.businesswire.com/news/home/ 20141219005468/en/SMI-Eye-Tracking-Glasses-Set-Industry-Standard)

CARROLL, M.; KOKINI, C.; MOSS, J. Training effectiveness of eye tracking-based feedback at improving visual search skills. International Journal of Learning Technology, 8(2), 147-168, 2013. https: //doi.org/10.1504/IJLT.2013.055671

CHANDRA, S.; SHARMA, G.; MALHOTRA, S.; JHA, D.; MITTAL, A. P. Eye tracking based human computer interaction: Applications and their uses. In 2015 International Conference on Man and Machine Interfacing (MAMI) (pp. 1-5). IEEE, 2015. https://doi.org/10.1109/MAMI. 2015.7456615

DEDKOVA, P.; POPELKA, S. Virtual 3D restoration of an extinct village and its eye-tracking assessment. Journal of Mobile Multimedia, 11(3-4), 181-192, 2015. (http://www.scopus.com/ inward/record.url?eid=2-s2.0-84949778007\&partnerlD=tZOtx3y).

ELMANSY, R. (2014) Empathic Design: The Most Difficult Simple Approach to Success-ful Design. Recuperado el 8 de Junio de 2015. Disponible en: http://www.designorate.com/empathicdesign-approach-to-successful-de-sign/ 
GIL, Antonio Carlos. Métodos e técnicas de pesquisa social. 6.ed. São Paulo: Atlas, 2011. GOBBI, A. G.; ANDRÉS, E.; MERINO, D.; SCHMIDT, G. Uso do eye tracking para obtenção de medidas quantitativas em testes de usabilidade: Um estudo focado na medida da satisfação. The use of eye tracking to obtain quantitative measurements in usability tests: a study focused on the measurement of the satis. Human Factors in Design, 6(11), 106-125, 2017.

GOLDSTEIN, S.; JOHNSTON, R.; DUFFY, J.; RAO, J.. The service concept: the missing link in service design researching?, In: Journal of Operations Management, n. 20, p. 121-134, 2002.

HASANZADEH, S.; ESMAEILI, B.; DODD, M. D. Measuring the Impacts of Safety Knowledge on Construction Workers' Attentional Allocation and Hazard Detection Using Remote EyeTracking Technology. Journal of Management in Engineering, 33(5), 1-17, 2017. https://doi.org/ 10.1061/(ASCE)ME.1943-5479.0000526

KOTLER, P; ARMSTRONG, G. Princípios de Marketing. Tradução: Vera Whately, revisão técnica: Roberto Meireles Pinheiro. 7. ed. Rio de janeiro: LTC, 1999.

LANGENBACH, M. L. Além do apenas funcional: inovação social e design de serviços na realidade brasileira. 2008. 123p. Dissertação de Mestrado em Ciências em Engenharia de Produção. Departamento de Engenharia. Universidade Federal do Rio de Janeiro. Brasil.

LOVELOCK, C.; WRIGHT, L. Serviços: Marketing e Gestão. São Paulo: Saraiva, 2003.

MAGER, B. Service Design. In: Erlhoff, Michael Marshale, Tim (ed.): Design Dictionary: Perspectives on Design Terminology. Basel: Birkhäuser, 2007.

MAGER, B. Service Design as an Emerging Field. In: S. Miettinen; M. Kivisto; Designing Services with Innovative Methods. Helsink: Helsinki Univesity of Art and Desig, 2009.

MANZINI, E. 2007. Sustainability: systemic change and social learning process. Disponível em: <http: / / www.producao.ufrj.br/design.isds/material.htm>. Acesso em: 28 nov. 2015.

MERINO, Giselle Schmidt Alves Díaz. GODP- Guia de Orientação para Desenvolvimento de Projetos: Uma metodologia de Design Centrado no Usuário. Florianópolis: Ngd/UFSC, 2016. Disponível em: ‘www.ngd.ufsc.br>. Acesso em: 01 abril. 2018.

PINE, B.; GILMORE, J., 1998, Welcome to the experience economy. Harvard Business Review, junho-agosto, p. 97-105.

PINHEIRO, T. The Service Startup: Design gets lean. United States: Amazon.com, 2014.

PRODANOV, C.C. Metodologia do trabalho científico: métodos e técnicas da pesquisa e do trabalho acadêmico. Novo Hamburgo: Feevale, 2013.

SCHMIDT, M. C.; MARTINS, D. G.; RODRIGUES, R. S.; GOBBI, A. G.; MERINO, G. S. A. D.; \& GOMES Ferreira, M. G. Desempenho do e-reader lev: uma análise utilizando a ferramenta eye tracking. Human Factors in Design, 5(10), 31-47, 2016. (http://revistas.udesc.br/index.php/ $\mathrm{hfd} /$ article/view/8833/6195)

STICKDORN, M. SCHNEIDER, J. (Orgs.). Isto é design thinking de serviços. Tradução de Mariana Bandarra. Porto Alegre: Bookman, 2014.

\section{AGRADECIMENTOS}

Agradecemos à Coordenação de Aperfeiçoamento de Pessoal de Nível Superior (CAPES), ao Conselho Nacional de Desenvolvimento Científico e Tecnológico (CNPq), à Fundação de Amparo à Pesquisa e Extensão Universitária (FAPEU), ao Programa UNIEDU PósGraduação, à Pró-Reitoria de Extensão e Assuntos Comunitários (Proex), à Rede de Pesquisa e Desenvolvimento de Tecnologia Assistiva (RPDTA) e ao Programa de PósGraduação em Design (POSDESIGN/UFSC). 